\title{
Mejora de engorde de cuyes (Cavia porcellus L.) a base de gramíneas y forrajeras arbustivas tropicales en la zona de Quevedo, Ecuador
}

\author{
Improved fattening guinea pig (Cavia porcellus L.) based on tropical forage grasses \\ and shrubs in Quevedo, Ecuador
}

\author{
Gary Alex Meza Bone 1, 2, 3, Rodrigo Paul Cabrera Verdezoto ${ }^{1,2 *}$, Jéssica Jessenia Morán Morán ${ }^{1,2}$, \\ Fabricio Fabián Meza Bone ${ }^{1}$, César Alberto Cabrera Verdesoto ${ }^{1,2}$, Calos Javier Meza Bone ${ }^{3}$, \\ Jéssica Sayonara Meza Bone ${ }^{2}$, María Gabriela Cabanilla Campos ${ }^{2}$, Francel Xavier López Mejía ${ }^{1}$, \\ José Luis Pincay Jiménez ${ }^{1}$, Tito Bohórquez Barros ${ }^{3}$, Juan Ortiz Dicado ${ }^{3}$
}

\section{RESUMEN}

La investigación se ejecutó en la Finca Experimental "La María”, propiedad de la Universidad Técnica Estatal de Quevedo (UTEQ), localizada en el kilómetro 7 1 1/2 de la Vía Quevedo-El Empalme, provincia de Los Ríos, cuya ubicación geográfica de $01^{\circ} 06^{\prime} 13^{\text {” de }}$ latitud sur y $79^{\circ} 29^{\prime} 22^{\prime \prime}$ de longitud oeste y a una altura de $73 \mathrm{msnm}$. La investigación tuvo una duración de 56 días. Los objetivos fueron: a) Determinar el incremento de peso en el engorde de cuyes a base de gramíneas tropicales. b) Determinar el incremento de peso en el engorde de cuyes a base de forrajeras arbustivas tropicales. c) Determinar la rentabilidad de los tratamientos. Se utilizaron 48 cuyes machos de 30 días de edad con un peso promedio de 411,11 g. La unidad experimental estuvo conformada por dos animales. Se aplicó un diseño de bloques completamente al azar (DBCA) con cuatro repeticiones. Para determinar diferencias entre medias de tratamiento se aplicó la prueba de rangos múltiples de Tukey ( $\mathrm{P} \leq 0,05)$. Se evaluó el consumo de forraje (g), consumo de alimento $(\mathrm{g})$, peso vivo $(\mathrm{g})$, ganancia de peso $(\mathrm{g})$, conversión alimenticia, peso a la canal (g) y el rendimiento a la canal (\%). La rentabilidad se determinó mediante la relación beneficio costo. El mayor consumo de forraje $(\mathrm{P}<0,01)$ lo registró el pasto saboya y la morera $\left(42,49\right.$ y $36,46 \mathrm{~g} \mathrm{MS}_{\text {animal }}^{-1} \mathrm{día}^{-1}$, respectivamente). El suministro de morera permitió incrementar $(\mathrm{P}<0,01)$ el peso vivo $(915,70 \mathrm{~g})$; la ganancia de peso $\left(9,06 \mathrm{~g}_{\text {animal }}^{-1} \mathrm{dí}^{-1}\right)$; conversión alimenticia más eficiente $(4,24)$; peso a la canal, rendimiento a la canal y la rentabilidad $(639,83 \mathrm{~g} ; 69,87 \%$ y $61,64 \%$, respectivamente). El uso de la cucarda en el engorde de cuyes mejorados afectan significativamente los principales índices productivos de esta especie.

Palabras clave: producción, cuyes, alimentación, gramíneas, arbustivas forrajeras tropicales.

\begin{abstract}
The investigation you executes in the Experimental Property "The María”, property of the State Technical University of Quevedo (UTEQ), located in the kilometer 7 1/2 of the Road Quevedo - The Connection, county of The Ríos whose geographical location of $1^{\circ} 6^{\prime} 13^{\prime \prime}$ of south latitude and 79 $29^{\prime} 22$ " of longitude west and to a height of $73 \mathrm{msnm}$. The investigation had duration of 56 days. The objectives were: to) to Determine the increment of weight in the one puts on weight of guinea pigs in base of gramineous tropical. b) to Determine the increment of weight in the one puts on weight of guinea pigs in base of forrajeras tropical arbustivas. c) to Determine the profitability of the treatments. 48 guinea males pigs were used without of 30 days of age with a weight average of $411.11 \mathrm{~g}$. The experimental unit was conformed by two animals. A design of blocks was applied totally at random (DBCA) with four repetitions. To determine differences among treatment stockings the test of ultiple ranges of Tukey it was applied $(P \leq 0.05)$. the forage consumption was evaluated $(\mathrm{g})$, I consummate of food $(\mathrm{g})$, I weigh I live $(\mathrm{g})$, gain of weight $(\mathrm{g})$, nutritious conversion, I weigh to the channel $(g)$ and the yield to the channel $(\%)$. The profitability determined it to him through the relationship I benefit cost. The biggest forage consumption $(P<0.01)$ I register it the grass saboya and the mulberry $\left(42.49\right.$ and $36.46 \mathrm{~g} \mathrm{MS} \mathrm{animal}^{-1}$ $d a y^{-1}$, respectively). The mulberry supply allowed to increase $(P<0.01)$ the alive weight $(915.70 \mathrm{~g})$; the gain of weight $(9.06$ $g$ animal $^{-1}$ day $\left.^{-1}\right)$; more efficient nutritious conversion (4.24); I weigh to the channel, yield to the channel and the profitability $(639.83 \mathrm{~g} ; 69.87 \%$ and $61.64 \%$, respectively). The use of the cucarda in the one puts on weight of improved guinea pigs they affect the main productive indexes of this species significantly.
\end{abstract}

Key words: production, guinea pigs, food, grasses, shrubs, tropical forage.

\footnotetext{
Instituto Tecnológico Superior Ciudad de Valencia. Los Ríos, Valencia, Ecuador.

Universidad Técnica Estatal de Quevedo. Los Ríos, Quevedo, Ecuador.

Universidad Técnica de Babahoyo Los Ríos, Babahoyo, Ecuador

* Autor para correspondencia: rpcabrera@uteq.edu.ec; gmeza@uteq.edu.ec
}

Fecha de Recepción: 28 Abril, 2014.

Fecha de Aceptación: 12 Junio, 2014. 


\section{Introducción}

El cuy es un pequeño roedor originario de los Andes, utilizado como alimento en una extensa región comprendida por Chile, Argentina, Bolivia, Perú, Ecuador y Colombia. Su crianza es generalizada en el ámbito rural como un animal de carne para autoconsumo, constituyéndose en una excelente alternativa para diversificar la dieta. Considerado por la ONU y la FAO como Una fuente de seguridad alimenticia de la población mundial de escasos recursos económicos (Sánchez et al., 2009).

En la cría y explotación de esta especie se descuidan aspectos importantes como una adecuada alimentación, manejo, higiene, etc., dando lugar a que no se alcancen buenos índices reproductivos y productivos. Es un herbívoro monogástrico, el cuy tiene dos tipos de digestión, una enzimática a nivel de estómago y otra microbial a nivel de ciego. Su mayor o menor actividad depende de la composición de la ración. Esto contribuye a darle versatilidad a los sistemas de alimentación.

La nutrición juega un rol importante en toda explotación pecuaria, el adecuado suministro de nutrientes conlleva a una mejor producción. El conocimiento de los requerimientos nutritivos de los cuyes nos permitirá poder elaborar raciones balanceadas que logren satisfacer las necesidades en cada una de las fases biológicas por las que atraviesa (gestación, lactancia, engorde).

El uso de leguminosas forrajeras arbóreas y arbustivas, de alto potencial productivo y alto valor nutritivo, es una de las diferentes alternativas que se plantean para mejorar la alimentación animal en las explotaciones pecuarias (Rodríguez y Guevara, 2002). La tendencia actual de utilizar forrajes de origen arbustivo o arbóreo se estimula por los incrementos de los precios de los granos de cereales y oleaginosas, lo que además de incrementar los costos de producción animal tiene el inconveniente que compiten con la alimentación humana.

Una de las razones que inducen al estudio de la explotación de cuyes es la necesidad de contribuir con la producción de carne a partir de una especie herbívora fácilmente adaptable a diferentes ecosistemas, en cuya alimentación se pueden utilizar insumos que no compiten con la alimentación del hombre y de otros animales monogástricos, pues estos pueden ser alimentados con forraje verde fresco por lo que se pone en consideración a la morera, caraca, cucarda, saboya, king grass y el maralfalfa, ya que son forrajes que se encuentran en nuestro medio para la alimentación animal.

\section{Materiales y Métodos}

La investigación se ejecutó en el Programa de Especies Menores de la Finca Experimental "La María", propiedad de la Universidad Técnica Estatal de Quevedo (UTEQ), localizado en el kilómetro $7^{1 / 2}$ de la vía Quevedo-El Empalme, provincia de Los Ríos. Situada entre las coordenadas geográficas $01^{0}$ 06 ' 13 " de latitud sur y $79^{0} 29^{\prime} 22$ " de longitud oeste, a una altura de $73 \mathrm{msnm}$, con una precipitación anual de $1.536,71 \mathrm{~mm} \mathrm{año}^{-1}$, temperatura promedio anual de $24,70{ }^{\circ} \mathrm{C}$, humedad relativa de $87,20 \%$, heliofanía media anual de 855,10 horas luz y topografía irregular.

En la fase de producción se aplicó un diseño de bloques completos al azar (DBCA), con cuatro repeticiones y la unidad experimental estuvo constituida por dos animales. Se aplicó la prueba de Tukey $(\mathrm{P} \leq 0,05)$, y para la valoración económica la Relación Beneficio-Costo. Se utilizaron 48 cuyes machos con un peso promedio de $411,11 \mathrm{~g}$, de 30 días de edad. Los factores bajo estudio durante la fase productiva fueron: Gramíneas (Saboya: Panicum maximun Jack; King-grass: Pennisetum purpureum $x$ Pennisetum typhoides y Maralfalfa: Pennisetum $s p)$ Arbustivas forrajeras tropicales (Morera: Morus alba; Caraca: Erythrina poeppigiana y Cucarda: Hibiscus rosa-sinensis) ad libitum.

En la etapa de producción las variables evaluadas fueron: consumo de forraje, consumo de balanceado, consumo de alimento, ganancia de peso, conversión alimenticia, peso y rendimiento a la canal.

\section{Resultados y Discusión}

El forraje de mayor consumo de forraje de gramíneas total $(\mathrm{P}<0,01)$ fue el pasto saboya $(42,49$ g animal ${ }^{-1}$ día $\left.^{-1}\right)$. Los valores obtenidos del pasto saboya, king-grass y maralfalfa sobre el consumo de forraje $\left(42,49 ; 41,07\right.$ y 40,05g MS animal ${ }^{-1}$ día $\left.^{-1}\right)$ son inferiores a los reportados por Sánchez et al. (2009), quienes al utilizar gramíneas tropicales en el engorde de cuyes mejorados sexados obtuvieron un consumo de 99,31 g MS animal ${ }^{-1}$ día $^{-1}$ para el saboya y a los de Yaringaño, 1994, quien al evaluar el efecto de cuatro raciones para cuyes en crecimiento obtuvo un consumo de forrajes de $124 \mathrm{~g}$. Pero cercanos a los reportados por Muscari et al. (1994), quienes al evaluar el engorde de cuyes 
con pasto elefante (Pennicetum purpureum) en la costa central obtuvieron consumos de 48,91 g MS animal $^{-1}$ día $^{-1}$. Debemos considerar que dependiendo del grado de aceptación del alimento, del aspecto nutritivo y principalmente de la composición nutritiva será el consumo que se produzca en cada una de las etapas fisiológicas de los animales, por lo tanto a mejor calidad menor cantidad y viceversa. Respecto del consumo de forrajeras arbustivas tropicales, la morera registra el mayor consumo total $(\mathrm{P}<0,01)$ y el menor valor la cucarda $(36,46$ y $18,43 \mathrm{~g}$ animal $^{-1} \mathrm{día}^{-1}$, respectivamente). Tabla 1. Estos consumos son inferiores a los reportados por Savón et al. 2006, quienes obtuvieron consumos para la morera y caraca de $56,00-55,50$ y 80,30 80,70 g MS animal ${ }^{-1}$ día $^{-1}$.

El consumo de balanceado total no registró diferencias estadísticas significativas $(\mathrm{P}>0,05)$, debido a que el suministro fue constante. Ver Cuadro1. Los valores obtenidos en la presente investigación $\left(13,20 \mathrm{~g} \mathrm{MS}\right.$ animal $^{-1}$ día $\left.^{-1}\right)$ son inferiores a los reportados por Yaringaño (1994), quien al evaluar el efecto de cuatro raciones para cuyes en crecimiento obtuvo un consumo de balanceado de 16,40 g MS animal $^{-1}$ día $^{-1}$. Al utilizar un concentrado como único alimento se requiere preparar una buena ración para satisfacer los requerimientos nutritivos de los cuyes. Bajo estas condiciones los consumos por animal ${ }^{-1}$ día $^{-1}$ se incrementan, pudiendo estar entre 40 a $60 \mathrm{~g} \mathrm{animal}^{-1}$ día $^{-1}$, esto dependiendo de la calidad de la ración.

En el consumo de alimentos los tratamientos a base de gramíneas no registraron diferencias estadísticas $(\mathrm{P}>0,05)$. Sin embargo se puede observar que el tratamiento a base de pasto saboya registró el mayor consumo de alimento (43,44 g MS animal ${ }^{-1}$ día $\left.^{-1}\right)$. Respecto del consumo de forrajeras arbustivas tropicales, la morera registra el mayor consumo de alimento $(\mathrm{P}<0,05)$ y el menor valor la cucarda $(37,41$ y $19,37 \mathrm{~g} \mathrm{animal}^{-1} \mathrm{día}^{-1}$, respectivamente). Tabla 1 . Cuando la alimentación del cuy es mixta, la proteína la obtiene por el consumo de la ración balanceada y el forraje; si es una forrajera, la respuesta en crecimiento es superior al logrado con gramíneas. La baja calidad de un forraje fuerza al animal a un mayor consumo de concentrado para satisfacer sus requerimientos. Los valores obtenidos para las gramíneas (saboya, king-grass y maralfalfa) sobre el consumo de alimento $(43,44 ; 42,01 ; 41,00 \mathrm{~g}$ MS animal $^{-1}$ día $^{-1}$ ) no concuerdan con lo indicado por Hurtado et al. (2012) y Sánchez et al. (2009) quienes sostienen que el máximo consumo de materia seca $(\mathrm{P}<0,05)$ obtenido en su investigación fue de $57,1 \mathrm{~g}$ MS animal ${ }^{-1}$ día $^{-1}$ al utilizar pasto india y $94,51 \mathrm{~g}$ MS animal ${ }^{-1}$ día $^{-1}$ al utilizar gramíneas tropicales en el engorde de cuyes mejorados, respectivamente.

Los valores obtenidos con las forrajeras arbustivas tropicales (morera, caraca y cucarda) sobre el consumo de alimento $(37,41 ; 33,37 ; 19,37$ g MS animal ${ }^{-1}$ día $^{-1}$, respectivamente) son inferiores a los señalados por Apráez et al. (2008), quienes al evaluar el efecto del empleo de forrajes y alimentos no convencionales sobre el comportamiento productivo, rendimiento a la canal y calidad de la carne de cuyes encontraron un consumo de alimento de 57,30 g MS animal ${ }^{-1}$ día $^{-1}$, estos niveles de consumos encontrados están dentro de lo indicado por Chauca. (1999); citado por Apráez et al. (2008), de 40 a 60 g MS animal ${ }^{-1}$ día $^{-1}$ cuando los cuyes consumen alimento concentrado de un alto valor nutricional.

La ganancia de peso registró diferencias estadísticas significativas $(\mathrm{P}<0,01)$, obteniendo el

Tabla 1. Engorde de cuyes mejorados (Cavia porcellus Linnaeus) con gramíneas y forrajeras arbustivas tropicales en la zona de Quevedo.

\begin{tabular}{lccccccccc}
\hline \multicolumn{1}{c}{ Factores } & $\begin{array}{c}\text { Consumo } \\
\text { forraje } \\
(\mathrm{g}) \mathrm{c}\end{array}$ & $\begin{array}{c}\text { Consumo } \\
\text { balanceado } \\
(\mathrm{g})\end{array}$ & $\begin{array}{c}\text { Consumo } \\
\text { alimento }\end{array}$ & $\begin{array}{c}\text { Peso final } \\
(\mathrm{g})\end{array}$ & $\begin{array}{c}\text { Ganancia } \\
\text { peso }(\mathrm{g}) .\end{array}$ & $\begin{array}{c}\text { Conversión } \\
\text { alimenticia }\end{array}$ & $\begin{array}{c}\text { Peso } \\
\text { canal }(\mathrm{g})\end{array}$ & $\begin{array}{c}\text { Rendimiento Rentabilidad } \\
\text { canal }(\%)\end{array}$ \\
\hline Saboya & $42,49 \mathrm{a}$ & $13,20 \mathrm{a}$ & $43,44 \mathrm{a}$ & $743,38 \mathrm{bc}$ & $6,82 \mathrm{bc}$ & $6,66 \mathrm{a}$ & $473,25 \mathrm{bc}$ & $64,27 \mathrm{bc}$ & 21,44 \\
King-grass & $41,07 \mathrm{ab}$ & $13,20 \mathrm{a}$ & $42,01 \mathrm{ab}$ & $814,60 \mathrm{ab}$ & $6,34 \mathrm{bc}$ & $6,79 \mathrm{a}$ & $529,50 \mathrm{~b}$ & $65,01 \mathrm{bc}$ & 41,91 \\
Maralfalfa & $40,05 \mathrm{ab}$ & $13,20 \mathrm{a}$ & $40,99 \mathrm{ab}$ & $812,13 \mathrm{ab}$ & $6,20 \mathrm{c}$ & $6,80 \mathrm{a}$ & $521,00 \mathrm{bc}$ & $64,08 \mathrm{bc}$ & 33,71 \\
Morera & $36,46 \mathrm{bc}$ & $13,20 \mathrm{a}$ & $37,41 \mathrm{bc}$ & $915,70 \mathrm{a}$ & $9,01 \mathrm{a}$ & $4,24 \mathrm{~b}$ & $639,83 \mathrm{a}$ & $69,87 \mathrm{a}$ & 61,64 \\
Caraca & $32,43 \mathrm{c}$ & $13,20 \mathrm{a}$ & $33,37 \mathrm{c}$ & $797,18 \mathrm{abc}$ & $8,06 \mathrm{ab}$ & $4,25 \mathrm{~b}$ & $540,25 \mathrm{ab}$ & $67,74 \mathrm{ab}$ & 39,30 \\
Cucarda & $18,43 \mathrm{~d}$ & $13,20 \mathrm{a}$ & $19,37 \mathrm{~d}$ & $664,18 \mathrm{c}$ & $4,43 \mathrm{~b}$ & $4,48 \mathrm{~b}$ & $416,75 \mathrm{c}$ & $62,76 \mathrm{c}$ & 10,83 \\
CV $(\%)$ & 7,27 & 00,00 & 7,08 & 7,95 & 11,08 & 9,48 & 8,97 & 2,84 & \\
\hline
\end{tabular}

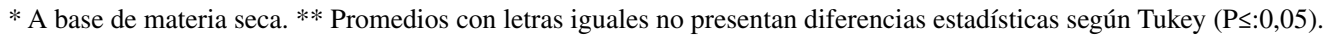


mayor valor el tratamiento a base de morera $(9,01 \mathrm{~g}$ animal $^{-1}$ día $^{-1}$ ). El comportamiento de los animales es diferente porque responden mejor cuando se les suministra una ración palatable como es el caso de las forrajeras arbustivas. Ver Tabla 1. Los valores obtenidos utilizando forrajeras arbustivas tropicales (morera, caraca y cucarda) sobre la ganancia de peso $\left(9,01 ; 8,06 ; 4,43 \mathrm{~g} \mathrm{animal}^{-1} \mathrm{día}^{-1}\right.$, respectivamente $)$ son inferiores a los encontrados por Apráez et al. (2008), quienes al evaluar el efecto del empleo de forrajes y alimentos no convencionales sobre el comportamiento productivo, rendimiento a la canal y calidad de la carne de cuyes encontró una ganancia media diaria g de 11,98. En tanto que Savón et al. (2006) obtuvieron incrementos de peso de 11,98 g animal $^{-1}$ día $^{-1}$ al utilizar concentrado y morera fresca. Estos valores de ganancia media diaria logrados se asemejan a los señalados por Apráez et al. (2008), quienes demostraron que animales alimentados con concentrado de alto valor nutricional pueden obtener ganancias medias diarias entre $10 \mathrm{y} 15 \mathrm{~g}$ animal $^{-1}$ día $^{-1}$; por otra parte, Yaringaño (1994), con el objetivo de evaluar el efecto de cuatro raciones para cuyes en crecimiento obtuvo ganancias de peso con caraca $5,7 \mathrm{~g}_{\text {animal }}{ }^{-1} \mathrm{día}^{-1}$.

Por otra parte Savón et al. (2006), al utilizar follaje de morera y concentrado, lograron incrementos de peso entre 9,3 y $9,7 \mathrm{~g}_{\text {animal }}{ }^{-1}$ día $^{-1}$, respectivamente, de forma similar Savón et al. (2006), al utilizar esta misma dieta encontraron incrementos de pesos de 9,5 $\mathrm{g} \mathrm{animal}^{-1}$ día ${ }^{1}$.

Los valores obtenidos del pasto saboya sobre la ganancia de peso $\left(6,82 \mathrm{~g}_{\text {animal }}{ }^{-1}\right.$ día $\left.^{-1}\right)$ son inferiores a lo reportado por Sánchez et al. (2009), quienes al utilizar gramíneas tropicales en el engorde de cuyes mejorados sexados obtuvieron $8,54 \mathrm{~g}$ animal $^{-1}$ día $^{-1}$, además con los de Proaño (1993), citado por Sánchez et al. (2009), quien al evaluar el efecto de la retama verde (Sphaero carpa) en sustitución $(0 ; 10 ; 20$ y $30 \%)$ de la alfalfa (Medicago sativa) sobre el sexo en las etapas de crecimiento y engorde (14-90 días) registró 7,34 $\mathrm{g}_{\text {animal }}{ }^{-1}$ día ${ }^{-1}$, además Sánchez et al. (2009) reportan que al evaluar el efecto del ramio (Bohemeria nivea Gaud), gramíneas y leguminosas en el engorde de cuyes logran 8,68 $\mathrm{g}_{\text {animal }}{ }^{-1}$ día $^{-1}$ para el tratamiento a base de pasto saboya + balanceado; sin embargo la ganancia se vio superada por la reportada por Cayancela (1993), quien al utilizar la harina de retama $(5 ; 10 ; 15$ y $20 \%$ de inclusión en la dieta) $9,65 \mathrm{~g}$ animal $^{-1}$ día $^{-1}$ con el $5 \%$ de inclusión y por los de Caycedo (1992), citado por Sánchez et al. (2009), quien reporta 10,0 g animal ${ }^{-1}$ día $^{-1}$.

Los resultados del pasto saboya reportados en la presente investigación obtenida para la ganancia de peso $\left(6,82 \mathrm{~g} \mathrm{animal}^{-1}\right.$ día $\left.^{-1}\right)$ son superiores a los reportados por Sánchez et al. 2009, quienes al evaluar el efecto del cubo nutricional suplementado con pasto saboya y elefante en el crecimiento y engorde de cuyes reportó 5,96 $\mathrm{g}$ animal ${ }^{-1}$ día $^{-1}$, para el tratamiento a base de pasto elefante + cubo multinutricional. De igual manera a los de Oleas, 1982 y Fernández (1984); citado por Sánchez et al. (2009), que obtuvieron ganancias de 6,11 y 6,43 g animal $^{-1}$ día $^{-1}$, respectivamente, utilizando diferentes niveles de gallinaza y quinuharina.

Los resultados del pasto king-grass en la presente investigación obtenida para la ganancia de peso $\left(6,34 \mathrm{~g}_{\text {animal }}{ }^{-1} \mathrm{día}^{-1}\right)$ son superiores a los reportados por Chacón et al., s/f., quienes al evaluar dos sistemas no convencionales de alimentación para cuyes alojados en piso registró ganancias de 3,66 $\mathrm{g}$ animal ${ }^{-1}$ día $^{-1}$. Además, con los de Meza, et al. (1994), quienes al evaluar el engorde de cuyes en la selva central encontró una ganancia de peso para el pasto elefante y brachiaria 5,86 y $5,30 \mathrm{~g} \mathrm{animal}^{-1}$ día ${ }^{-1}$, discrepando con los valores reportados por Muscari et al. (1994), quienes al evaluar en el engorde de cuyes con pasto elefante (Pennicetum purpureum) en la costa central obtuvieron ganancias de $8,10 \mathrm{~g}_{\text {animal }}{ }^{-1} \mathrm{dí}^{-1}$. Respecto de la conversión alimenticia, los tratamientos a base de forrajeras arbustivas tropicales (morera, caraca, cucarda) registraron las conversiones alimenticias más eficientes, en relación a las gramíneas (saboya, king grass, maralfalfa), estableciéndose diferencias estadísticas significativas $(\mathrm{P}<0,05)$. Ver Tabla 1 .

Las conversiones registradas utilizando gramíneas en la presente investigación $(6,66 ; 6,79$ y 6,80 , respectivamente) son más eficientes que las reportadas por Sánchez et al. (2009), quienes al utilizar gramíneas tropicales en el engorde de cuyes mejorados sexados obtuvieron conversiones de 13,8 (Cevallos, 1995 y Padilla, 1995); citado por Sánchez et al. (2009), quienes reportan conversiones de 12,6 y 7,02, respectivamente.

Los resultados del pasto king-grass obtenidos en la presente investigación $(6,79)$ fue más eficiente que los reportados por Chacón et al. s/f., quienes sostienen que al evaluar dos sistemas no convencionales de alimentación para cuyes alojados en piso registraron una conversión de 11,93 y con los de Caycedo, 
(1992); citado por Chacón et al. s/f. quien reporta conversiones de 7 a 12, al alimentar a cuyes a base de forraje; Meza et al. (1994) indica que al evaluar el engorde de cuyes en la selva central encontraron conversiones para el pasto king-grass; elefante y brachiaria 8,$32 ; 10,93 ; 12,12$, respectivamente. Por otra parte Muscari et al. (1994) evaluaron el engorde de cuyes con pasto elefante (Pennicetum purpureum) en la costa central obteniendo una conversión de 6,04.

Los resultados en la presente investigación utilizando forrajeras arbustivas tropicales concuerdan con las reportadas por Apráez et al. (2008), quienes manifiestan que al evaluar el efecto del empleo de forrajes y alimentos no convencionales sobre el comportamiento productivo, rendimiento a la canal y calidad de la carne de cuyes encontró una conversión alimenticia de 4,78, para la morera, y con Moncayo (1999), citado por Apráez et al. (2008), quienes indican que la conversión alimenticia oscila entre 4 y 7 , cuando a los cuyes se les suministra alimento concentrado de alto valor proteico y energético. Pero más eficientes que los reportados por Yaringaño (1994), quien con el objetivo de evaluar el efecto de cuatro raciones para cuyes en crecimiento registró conversiones de 7,5 y 7,91, respectivamente.

El mayor rendimiento a la canal $(\mathrm{P}<0,01)$ lo registró el tratamiento a base de morera $(69,87 \%)$ y el menor con el tratamiento a base de cucarda $(62,76 \%)$. Sin embargo se puede observar que entre los tratamientos a base de gramíneas no se registran diferencias estadísticas significativas $(\mathrm{P}>0,05)$. Ver Tabla 1. Esta mejor respuesta refleja el comportamiento general de los animales y que tienen como consecuencia la relación directa entre el peso final y la ganancia de peso, por lo tanto la individualidad genética, la conversión alimenticia y el manejo en general al que son sometidos los cuyes nos darán como respuesta un excelente rendimiento a la canal.

Los valores obtenidos del pasto king-grass, saboya y maralfalfa sobre el rendimiento a la canal $(65,01 ; 64,27$ y $64,08 \%$, respectivamente) son semejantes a lo reportado por Sánchez y col.
2009, quienes al utilizar gramíneas tropicales en el engorde de cuyes mejorados sexados obtuvieron un rendimiento del $65,2 \%$, pero inferiores a los señalados por Padilla (1990), citado por Sánchez et al. (2009), sostiene que al evaluar niveles de gallinaza en la alimentación de cuyes en las fases de gestación, lactancia, crecimiento y engorde registró rendimientos del obtuvieron 68,01\% y a los de Cevallos (1995); Ante 2002; Plaza y Suárez, (2001), citados por Sánchez et al. (2009), registraron rendimientos del 72,54; 67,50 y 71,64\%, respectivamente.

Los resultados registrados en la presente investigación para la morera $(69,87 \%)$ superan a los mencionados por Apráez et al. (2008), que al evaluar el efecto del empleo de forrajes y alimentos no convencionales sobre el comportamiento productivo, rendimiento a la canal y calidad de la carne de cuyes obtuvieron $65,20 \%$. Además superan a los de Mercado et al. (1994); citado por Apráez et al. (2008), que al estudiar el rendimiento de la canal en cuyes cebados con alimentos concentrados de alto valor nutricional obtuvieron valores entre 62 y $68 \%$ de rendimiento a la canal.

La mayor rentabilidad la registró el tratamiento a base de morera seguido de king grass y caraca $(61,64 ; 41,91$ y $39,30 \%$, respectivamente). Tabla 1 .

\section{Conclusiones}

El mayor consumo de forraje y consumo de alimento total se obtiene al suministrar pasto saboya en el engorde de cuyes en la zona de Quevedo.

La forrajera arbustiva tropical de mayor consumo en el engorde de cuyes mejorados en la zona de Quevedo fue la morera.

El mayor peso vivo, ganancia de peso, conversión alimenticia, peso a la canal, rendimiento a la canal $\mathrm{y}$ rentabilidad se obtiene al suministrar morera en el engorde de cuyes mejorados en la zona de Quevedo.

Al suministrar cucarda se ven afectados los principales parámetros productivos en el engorde de cuyes mejorados en la zona de Quevedo. 


\section{Literatura Citada}

Albert, A.; Vera, M.; Savón, L.

2006. Digestibilidad de nutrientes de las especies Trichantera gigantea (H \& B) (nacedero) Morus alba Lin. (Morera) y Erythrina poe-ppigiana (walp. O.F.) (Piñón) para la alimentación de cuyes (Cavia porcellus). In: IV Congreso Latinoamericano de Agro-forestería para la Producción. Pecuaria Sostenible. Varadero, Cuba.

Apráez, J.; Fernández, L.; Hernández, A.

2008. Efecto del empleo de forrajes y alimentos no convencionales sobre el comportamiento productivo, rendimiento en canal y calidad de la carne de cuyes (Cavia porcellus). Artículo de investigación Universidad de Caldas Veterinaria y Zootecnia. vet.zootec. 2 (2): 29-34.

Cayancela, A.

1993. Efecto de varios niveles de harina de retama en la alimentación de cuyes en las etapas de crecimiento-engorde. IV Congreso Latinoamericano de Cuyecultura. Riobamba, Ecuador. pp. 35-37.

Chacón, E.; Velázquez, F.; Barrera, H.

s/f. Evaluación no convencionales de la alimentación para cuyes alojados en pienso. Granma-Cuba. edy@udg.co.cu Consultado el 11/12/2013.

Hurtado, D.; Nocua, S.; Narváez, W.; Vargas, J.

2012. Valor nutricional de la morera (Morus sp.), matarratón (Gliricidia sepium), pasto india (Panicum máximum) y arboloco (Montanoa quadrangularis) en la alimentación de cuyes (Cavia porcellus). Artículo de investigación Universidad de Caldas Veterinaria y Zootecnia. vet.zootec. 6 (1): 56-65.

Meza, J.; Roman, N.; Hovisco, M.

1994. Engorde de Cuyes en la Selva Central. Investigaciones en Cuyes. Instituto Nacional de Investigación Agraria (INIA)
Centro Internacional de Investigaciones para el Desarrollo. Resúmenes. En. Chauca L., (ed). Lima, Perú. Pp. 141.

Muscari, J.; Vásquez, F.; Chauca, L.

1994. Instituto Nacional de Investigaciones Agraria Engorde de cuyes compasto elefante (Pennicetum purpureum) en la costa central. UNA La Molina, Estación Experimental Agropecuaria La Molina. Lima, Perú. Pp. 25 Disponible (en Línea). http:// idl-bnc.idrc.ca/dspace/bitstream/10625/14460/1/101868. pdf. Consultado el 05/02/2014.

Sánchez, A.; Sánchez, S.; Godoy, S.; Díaz, R.; Vega, N.

2009. Gramíneas tropicales en el engorde de cuyes mejorados sexados (Cavia porcellus linnaeus) en la zona de la Maná. Revista Ciencia y Tecnología. Ecuador. 2: 25-28.

Savón, L.; Ly, L.; Albert, Dihigo L.

2006. Avances en el uso de follaje de morera en la alimentación de especies monogástricas. In; IV Congreso Latinoamericano de Agro-foresteria para la producción Pecuaria Sostenible. Varadero, Cuba.

Rodríguez, I.; Guevara, E.

2002. Producción de materia seca y valor nutritivo de la leguminosa arbustiva cratylia argentea en el sur del estado Anzoategui, Venezuela, producción de materia seca y valor nutritivo de la leguminosa arbustiva cratylia argentea en el sur del estado Anzoategui, Revista Científica Vol. XIISuplemento 2, octubre, 589-594, 2002.

Yaringaño, C.

1994. Comparativo de cuatro raciones para cuyes (Cavia cobayo) en crecimiento. En Chauca L., (ed). Investigaciones en cuyes. Instituto nacional de Investigaciones Agrarias (INIA), Lima, Perú. Pp. 56-57. 